\title{
Sca1 marks a reserve endothelial progenitor population that preferentially expand after injury
}

\author{
Juan Tang ${ }^{1}$, Huan Zhu ${ }^{1}$ ', Shaoyan Liu², Haixiao Wang ${ }^{1}$, Xiuzhen Huang ${ }^{1}$, Yan Yan², Lixin Wang ${ }^{2}$ and \\ Bin Zhou (iD) $1,3,4,5 \bowtie$
}

Dear Editor,

Vascular endothelial cell renewal, repair and regeneration are critical for tissue homeostasis and response to injuries ${ }^{1}$. Unraveling the heterogeneity and hierarchy of endothelial cells in homeostasis and after injuries provides valuable information of potential targets for therapeutic neovascularization. Stem cell antigen-1 (Sca1) is a member of the ly- 6 family, which was reported as cell surface markers of hematopoietic stem cells ${ }^{2}$. Sca1 ${ }^{+}$progenitor cells residing in the heart do not contribute to cardiomyocytes, but instead adopt vascular endothelial cell fate ${ }^{3-5}$. Whether Sca1-expressing cells represent a unique population of endothelial cells (ECs) that differs from other Sca1 ${ }^{-}$ECs remains largely unknown. In addition, whether the Scal expression heterogeneity in ECs may indicate the existence of a functional hierarchy for endothelial cells in angiogenesis is unclear. We first isolated $\mathrm{CD}^{+} 1^{+}$endothelial cells by FACS and performed single-cell RNA sequencing (scRNA-seq). Uniform manifold approximation and projection (UMAP) analysis of this dataset revealed clusters of endothelial and adventitial cell populations based on marker gene expression (Supplementary Fig. S1a, b), with Ly6a (Sca1) marking different populations of ECs and makers for others subtype cells such as pericytes, endocardial cells and fibroblasts (Supplementary Fig. S1b, c). Further pathway enrichment analysis showed that cell proliferation and angiogenesis-related pathways were highly enriched in Scal $1^{\text {high }}$ ECs compared with the Scal ${ }^{\text {low }}$ ECs

\footnotetext{
Correspondence: Bin Zhou (zhoubin@sibs.ac.cn)

${ }^{1}$ The State Key Laboratory of Cell Biology, CAS Center for Excellence on Molecular Cell Science, Shanghai Institute of Biochemistry and Cell Biology, Chinese Academy of Sciences, University of Chinese Academy of Sciences, Shanghai, China

${ }^{2}$ Zhongshan Hospital, Fudan University, Shanghai, China

Full list of author information is available at the end of the article

These authors contributed equally: Juan Tang, Huan Zhu
}

(Supplementary Fig. S1d), indicating these Sca1 ${ }^{\text {high }}$ ECs may exhibit specific functions during cardiac homeostasis and after injuries. Next we generated Sca1-2A-CreER;R26$G F P^{6}$ to lineage trace $\mathrm{Sca}^{+}$cells in the adult tissues during homeostasis and after injury (Fig. 1a). We collected heart samples at 24-48 hours after tamoxifen induction (Fig. 1b). Immunostaining for GFP, VE-Cad or PECAM showed that $30.54 \pm 1.01 \%$ of $\mathrm{VE}-\mathrm{Cad}^{+}$and $31.90 \pm 0.67 \%$ of PECAM ${ }^{+}$ECs express GFP (Fig. 1c, d). We confirmed that $\sim 30 \%$ of ECs were $\mathrm{GFP}^{+}$by FACS analysis of heart ventricles (Fig. 1e). We next examined the heart tissues at 12 to 16 weeks after tamoxifen treatment (Fig. 1f). By immunostaining and FACS analysis of heart ventricles, we did not find a significant increase of GFP percentage in ECs after 12-16 weeks' tracing (Fig. 1g-i). EdU incorporation assays showed there was no significant difference of the percentage of EdU ${ }^{+}$ECs between $\mathrm{GFP}^{-}$and $\mathrm{GFP}^{+}$ EC populations (Fig. 1j). These data indicate that $\mathrm{Sca}^{+}$ ECs proliferate at the similar rate as $\mathrm{Scal}^{-} \mathrm{ECs}$ at homeostasis.

We next exposed Sca1-2A-CreER;R26-GFP mice to cardiac stress by transverse aortic constriction (TAC) model at two weeks after tamoxifen treatment (Fig. 1k). Sirius red staining on heart sections showed more fibrosis after TAC (Fig. 1l). Immunostaining for GFP, VE-Cad or PECAM on tissue sections showed a significant increase of the percentage of $\mathrm{GFP}^{+}$ECs in TAC group compared with Sham (Sham $29.34 \pm 0.98 \%$ vs TAC $59.12 \pm 1.85 \%$ of VE-Cad ${ }^{+}$ECs; Sham $30.82 \pm 1.48 \%$ vs TAC $58.64 \pm 1.04 \%$, Fig. $1 \mathrm{~m}$ ). Flow cytometric analysis of left ventricle confirmed that the percentage of $\mathrm{GFP}^{+} \mathrm{ECs}$ in TAC was significantly higher than sham group (Sham $30.05 \pm 1.02 \%$ vs TAC $57.69 \pm 0.96 \%$, Fig. 1n). By detection of EdU incorporation, we found more $\mathrm{GFP}^{+} \mathrm{ECs}$ incorporate EdU than GFP $^{-}$ECs after TAC (Fig. 1o). This result was confirmed by Ki67 and pHH3 staining (Supplementary 


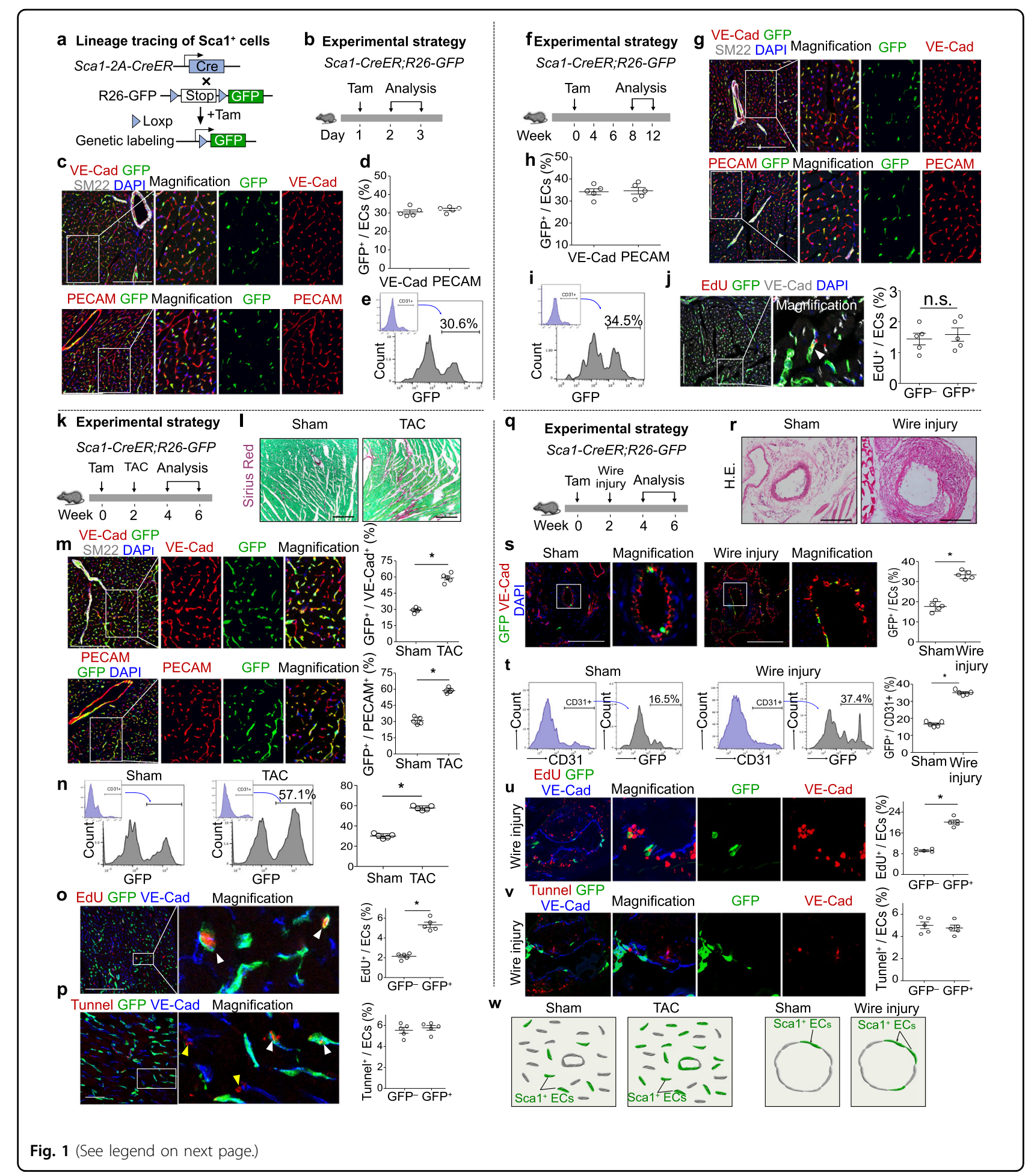

Fig. S2a, b). In addition, there was no significant difference in cell death between $\mathrm{Sca}^{+}$and Sca1 ${ }^{-}$ECs in post-injury hearts (Fig. 1p). Taken together, these data demonstrate that $\mathrm{Sca}^{+}$ECs respond to cardiac stress and expand more preferentially after injury.
To examine if Sca1 ${ }^{+}$ECs in the large arteries are also unique in cell proliferation compared with $\mathrm{Sca}^{-}$ECs, we performed wire-induced femoral artery injury model on Sca1-2A-CreER;R26-GFP mice at two weeks after tamoxifen treatment (Fig. 1q). HE staining showed neointimal 
(see figure on previous page)

Fig. $1 \mathrm{Sca} 1^{+}$endothelial cells expand preferentially after injuries. a Schematic figure showing genetic lineage tracing of Sca ${ }^{+}$cells. b Schematic figure showing experimental design. c Immunostaining for GFP, VE-Cad, SM22, or PECAM on heart sections. d Quantification of the percentage of VE-Cad ${ }^{+}$ or PECAM ${ }^{+}$endothelial cells (ECs) expressing GFP. e Flow cytometric analysis of CD31 ${ }^{+}$ECs expressing GFP. $\mathbf{f}$ Schematic diagram showing experimental design for homeostasis study. $\mathbf{g}$ Immunostaining for GFP, VE-Cad, SM22, or PECAM on heart sections. $\mathbf{h}$ Quantification of the percentage of VE-Cad ${ }^{+}$or $\mathrm{PECAM}^{+}$ECs expressing GFP. $\mathbf{i}$ Flow cytometric analysis and quantification of the percentage of $\mathrm{CD} 31^{+}$cells expressing GFP. $\mathbf{j}$ Immunostaining for EdU, GFP, and VE-Cad on heart sections. Quantification of the percentage of EdU ${ }^{+}$cells in $\mathrm{GFP}^{+}$or GFP ${ }^{-}$populations. $\mathbf{k}$ Schematic diagram showing experimental design for TAC study. I Sirius red staining on sections from sham and TAC hearts. $\mathbf{m}$ Immunostaining for GFP, VE-Cad, SM22, or PECAM on heart sections, and the quantification of the percentage of VE-Cad ${ }^{+}$or PECAM ${ }^{+}$ECs expressing GFP. $\mathbf{n}$ Flow cytometric analysis and quantification of the percentage $\mathrm{CD}_{3} 1^{+}$ECs expressing GFP. o Immunostaining for EdU, GFP, and VE-Cad on TAC heart sections. Quantification of the percentage of GFP' or GFP' ${ }^{+}$ECs incorporating EdU. p Immunostaining for TUNEL, GFP and VE-Cad on TAC heart sections. Quantification of the percentage of GFP- or GFP+ ECs that are TUNEL $^{+}$. q Schematic diagram showing experimental design for wire-induced injury. $\mathbf{r}$ HE staining on artery sections from sham or wire injury groups. $\mathbf{s}$ Immunostaining for GFP and VE-Cad on sections. Quantification of the percentage of ECs expressing GFP. $\mathbf{t}$ Flow cytometric analysis and quantification of the percentage of CD31 $1^{+}$ECs expressing GFP. $\mathbf{u}$ Immunostaining for EdU, GFP, and VE-Cad on injury arteries. Quantification of the percentage of GFP- or $\mathrm{GFP}^{+}$ECs incorporating EdU. v Immunostaining for TUNEL, GFP, and VE-Cad on injury arteries. Quantification of the percentage of GFP' or GFP $^{+}$ECS that $^{-}$ are TUNEL ${ }^{+} . \mathbf{w}$ Cartoon image showing preferential expansion of Sca1 ${ }^{+}$ECs after TAC or wire induced artery injuries. Scale bars, $100 \mu \mathrm{m}$. Data are means \pm $\mathrm{SEM} ; n=5 ;{ }^{*} P<0.05 ; \mathrm{ns}$, non-significant. Each figure is representative of 5 individual biological samples.

formation, indicating successful wire-induced vessel injury (Fig. 1r). Immunostaining for GFP and VE-Cad on tissue sections showed a significant increase of the percentage of $\mathrm{GFP}^{+}$ECs after wire-induced injury compared with sham group (Sham $17.63 \pm 1.07 \%$ vs Wire injury $33.37 \pm 0.88 \%$, Fig. 1s). Flow cytometric analysis showed that a significant increase of $\mathrm{GFP}^{+}$ECs percentage after wire injury (Sham $16.66 \pm 0.62 \%$ vs Wire injury $34.97 \pm 0.55 \%$, Fig. 1 t). By EdU incorporation analysis, we found the percentage of $\mathrm{GFP}^{+}$ECs incorporating EdU was significantly higher than that of $\mathrm{GFP}^{-}$ECs after wire injury (Fig. 1u). In addition, the percentage of $\mathrm{GFP}^{+}$ECs expressing Ki67 or pHH3 was significantly higher than that of $\mathrm{GFP}^{-} \mathrm{ECs}$ in injured arteries (Supplementary Fig. S2c, d). We did not detect any significant difference in cell death between $\mathrm{Scal}^{+}$and Sca1 $^{-}$ECs after wire injury (Fig. 1v). Taken together, $\mathrm{Sca}^{+}$ECs in the large arteries also respond and expand preferentially after injury.

This study reported that $\mathrm{Scal}^{+}$ECs represent a distinct EC sub-population that preferentially expand after TAC or wire injury (Fig. 1w). Recently, a number of reports have suggested that $\mathrm{Sca}^{+}$multipotent stem cells can give rise to endothelial cells. Qu-Petersen et al. showed that $\mathrm{Sca}^{+}{ }^{+}$skeletal muscle-derived stem cells are able to differentiate into endothelial cells, and contribute to the regeneration of the skeletal muscle in a murine model of Duchenne's muscle dystrophy ${ }^{7}$. Myocardium-derived adult $\mathrm{Sca}^{+}$cells isolated by cell sorting techniques were also capable of differentiating into endothelial cells $^{8-10}$. However, there is no direct in vivo evidence supporting the existence of $\mathrm{Sca}^{+}$side population cells for endothelial cell contribution during tissue homeostasis and after injury. In our study, we used Sca1-CreER to fatemap Sca1 ${ }^{+}$cells in tissue homeostasis and after injuries. By scRNA-seq, we found cell proliferation or cell cycle regulation and angiogenesis-related pathways were highly enriched in Sca1-high ECs compared with that of Sca1low ECs, indicating the potential higher cell proliferation and angiogenesis-related function of these cells (Supplementary Fig. 1d). While we found that there is no significant difference of EC renewal between $\mathrm{Sca}^{+}$and $\mathrm{Sca}^{-}$EC populations under homeostasis by EDU/Ki67 and $\mathrm{pHH} 3$ immunostaining. Consistent with results in heart after TAC injury, we found the percentage of Sca1 ${ }^{+}$ ECs that have incorporated EdU was significantly higher than that from $\mathrm{Sca1}^{-} \mathrm{ECs}$ after wire injury. These data suggested that $\mathrm{Sca}^{+}$ECs have stronger proliferation ability, which is activated after injury. The detection of cell proliferation difference in scRNA-seq but not by immunostaining during homeostasis could be explained by the sensitivity of the two different techniques in measuring gene expression. Our fate mapping results suggest that $\mathrm{Sca}^{+}$cells represent a reserve endothelial cell population that preferentially expands after injuries. Our study also supported the existence of an endothelial cell hierarchy within tissue, providing new avenues for future therapeutic interventions for vascular diseases. While Sca1 ${ }^{+}$ endothelial progenitors give rise to more endothelial cells after injuries, whether other endothelial cell populations or progenitors contribute to new endothelial cells need further studies. Future studies would be required to unravel the underlying mechanism for contribution of $\mathrm{Sca}^{+}{ }^{+}$cells to more endothelial cells in vascular repair and regeneration.

\section{Acknowledgements}

This work was supported by National Key Research \& Development Program of China (2019YFA01 10403, 2018YFA0107900, 2019YFA0802000, 2018YFA0108100, 2019YFA0802803, 2020YFA0803202), National Science Foundation of China (82088101, 31801215, 32070727), Yong Elite Scientist Sponsorship Program by CAST (YESS, 2018QNRC001), China Postdoctoral Science Foundation, Sanofi-SIBS Fellowship, AstraZeneca and Royal SocietyNewton Advanced Fellowship.

\footnotetext{
Author details

${ }^{1}$ The State Key Laboratory of Cell Biology, CAS Center for Excellence on Molecular Cell Science, Shanghai Institute of Biochemistry and Cell Biology, Chinese Academy of Sciences, University of Chinese Academy of Sciences, Shanghai, China. ${ }^{2} Z$ hongshan Hospital, Fudan University, Shanghai, China.
} 
${ }^{3}$ School of Life Science and Technology, ShanghaiTech University, Shanghai, China. ${ }^{4}$ School of Life Science, Hangzhou Institute for Advanced Study, University of Chinese Academy of Sciences, Hangzhou, China. Institute for Stem Cell and Regeneration, Chinese Academy of Sciences, Beijing, China

\section{Author contributions}

J.T., H.Z., S.L., H.W., X.H., Y.Y., and L.W. bred the mice, performed experiment, analyzed data, provided valuable comments, or edited manuscript. B.Z. supervised the study, analyzed the data and wrote the manuscript. All authors reviewed the manuscript.

\section{Competing interests}

The authors declare no competing interests.

\section{Publisher's note}

Springer Nature remains neutral with regard to jurisdictional claims in published maps and institutional affiliations.

Supplementary information The online version contains supplementary material available at https://doi.org/10.1038/s41421-021-00303-z.

Received: 29 October 2020 Accepted: 30 June 2021

Published online: 28 September 2021

\section{References}

1. Gomez-Salinero, J. M. \& Rafii, S. Endothelial cell adaptation in regeneration Science 362, 1116-1117 (2018).

2. van de Rijn, M., Heimfeld, S., Spangrude, G. J. \& Weissman, I. L. Mouse hematopoietic stem-cell antigen Sca-1 is a member of the Ly- 6 antigen family. Proc. Natl. Acad. Sci. USA 86, 4634-4638 (1989).

3. Vagnozzi, R. J. et al. Genetic lineage tracing of Sca-1(+) cells reveals endothelial but not myogenic contribution to the murine heart. Circulation 138, 2931-2939 (2018)

4. Soonpaa, M. H. et al. Absence of cardiomyocyte differentiation following transplantation of adult cardiac-resident Sca-1(+) cells into infarcted mouse hearts. Circulation 138, 2963-2966 (2018).

5. Lee, R. T. Adult cardiac stem cell concept and the process of science. Circulation 138, 2940-2942 (2018).

6. Tang, J. et al. Fate mapping of Sca1 $(+)$ cardiac progenitor cells in the adult mouse heart. Circulation 138, 2967-2969 (2018).

7. Qu-Petersen, Z. et al. Identification of a novel population of muscle stem cells in mice: potential for muscle regeneration. J. Cell Biol. 157, 851-864 (2002).

8. Beltrami, A. P. et al. Adult cardiac stem cells are multipotent and support myocardial regeneration. Cell 114, 763-776 (2003).

9. Oh, $\mathrm{H}$. et al. Cardiac progenitor cells from adult myocardium: homing, differentiation, and fusion after infarction. Proc. Natl. Acad. Sci. USA 100 12313-12318 (2003).

10. Pfister, O. et al. CD31- but Not CD31+ cardiac side population cells exhibit functional cardiomyogenic differentiation. Circ. Res. 97, 52-61 (2005). 